\title{
Synthesis of Pyrrolo[2,3-b]quinolines by Palladium-catalyzed Heteroannulation
}

\author{
Moon Bae Gee, Won Jung Lee, and Eul Kgun Yum" \\ Department of Chemistry; Chmonam National Liversity, Yusung, Daejeon 305-764. Korea \\ Received April 21, 2003
}

\begin{abstract}
Palladium-catalyzed heteroannulation of 2-amino-3-jodoquinoline derivatives and 1-trimethylsilyl internal alkynes provided highly regioselective pyrrolo[2,3-b]quinolines with trimethylsilyl group next to the nitrogen atom in the pycrole ring.
\end{abstract}

Key Words : Pyrrolo[2,3-b] quinolines, ]alladium, Internal alkynes, Heteroannulation

\section{Introduction}

Pytrolo[2.3-b]quinolines are chemically interesting molecules due to their structural similarity to furo[2,3-b]quinolines, which occur widely in natural biologically active products. ${ }^{1}$ Pyrrolo[2,3-b]quinolines have also a wide variety of biological activities, including anti-inflammatory, anticonvulsant, antihypertensive, antipyretic, analgesic, anti-MDR, and anticancer activity. ${ }^{2}$ Many synthetic methods for pyrrolo[2,3-b]quinolines $^{\text {2a }}$ have been reported, including pyrolysis of azepine derivatives, ${ }^{3}$ photolysis of 3-(2-aminobenzylidene)pyrrolidin-2(1H)-ones, ${ }^{\prime}$ cyclization of 2-chloro-3-(2-chloroethyl)-quinoline." cyclization of $N$-phenyl-3-aminopropiolamides, ${ }^{6}$ reaction of 3 -(lithiomethyl)quinoline with nitriles, ${ }^{7}$ and the aza-Wittig/electrocyclic ring-closure nitrene insertion process. ${ }^{8}$ However, only a few synthetic methods have been described for totally aromatic $1 H$-pyrrolo[2,3-b]quinolines with limited substituents. ${ }^{7-9}$ Larock and coworkers reported a palladium-catalyzed intermolecular reaction of $o$-haloarylalnine and internal alkynes to give indoles in one operation. ${ }^{30}$ The heteroannulation method could be an effective synthetic procedure for preparing a variety of heterocycles. " Therefore, we applied the synthetic method to the synthesis of heterocyclic azaindoles ${ }^{12}$ and pyrrolo[3.2-c] quinolines. ${ }^{1.3}$ Here, we report the convenient synthesis of 2,3-disubstituted pyrrolo[2,3-h] quinolines using palladium-catalyzed heteroannulation.

\section{Results and Discussion}

The 2-chloro-3-iodoquinoline was prepared by regioselective lithiation of 2-chloroquinoline with LDA followed by treatment with $I_{2}$ as an electrophile to prepare starting materials for palladium-catalyzed heteroannulation. The substitution reaction of 2-chloro-3-iodoquinoline with the corresponding amines afforded $\mathrm{N}$-alkyl or aryl 2-amino-3jodoquinolines in $70-80 \%$ yields. ${ }^{14}$ Initially, we optimized the reaction of 2-methylamino-3-iodoquinoline and 1trimethylsilyl propyne with various palladium species and bases. The results are summarized in lable 1 . We first examined the effect of different palladium species on the product yield using KOAc as the base and DMF as the solvent. The reaction using $\mathrm{Pd}(\mathrm{dba})_{2}$ or $\mathrm{Pd}(\mathrm{OAc})_{2}$ as the
Table I. Optimization of palladium-catalyzed heteroannulation for py rolo $[2,3-b]$ quinolines

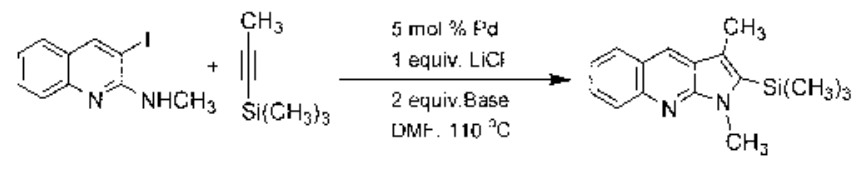

\begin{tabular}{cllcc}
\hline Fintry & $\begin{array}{c}\text { Palladium } \\
\text { Source }\end{array}$ & Base & $\begin{array}{c}\text { Ruaction time } \\
(\mathrm{h})\end{array}$ & $\begin{array}{c}\text { lsolated yiclds } \\
(\%)\end{array}$ \\
\hline 1 & $\mathrm{Pd}(\mathrm{dba})_{2}$ & $\mathrm{KOAc}$ & 14 & 65 \\
2 & $\mathrm{Pd}\left(\mathrm{PPh}_{3}\right)_{2} \mathrm{Cl}_{2}$ & $\mathrm{KO \Lambda c}$ & 14 & 54 \\
3 & $\mathrm{Pd}\left(\mathrm{PPh}_{3}\right)_{2}$ & $\mathrm{KO \Lambda c}$ & 20 & 50 \\
4 & $\mathrm{Pd}(\mathrm{OAc})_{2}$ & $\mathrm{KO \Lambda c}$ & 10 & 65 \\
5 & $\mathrm{Pd}(\mathrm{OAc})_{2}$ & $\mathrm{LiONc}_{2}$ & 10 & 75 \\
6 & $\mathrm{Pd}(\mathrm{OAc})_{2}$ & $\mathrm{Na}_{2} \mathrm{CO}_{3}$ & 10 & 50 \\
7 & $\mathrm{Pd}(\mathrm{OAc})_{2}$ & $\mathrm{~K}_{2} \mathrm{CO}_{3}$ & 10 & 60 \\
8 & $\mathrm{Pd}(\mathrm{OAc})_{2}$ & $\mathrm{Cs}_{2} \mathrm{CO}_{3}$ & 10 & 67 \\
9 & $\mathrm{Pd}(\mathrm{OAc})_{2}$ & $\mathrm{Ct}_{3} \mathrm{~N}$ & 10 & 60 \\
\hline
\end{tabular}

"Actual amounts of reagents uscd: $0.5 \mathrm{mmol}$ aryl halide. $1.0 \mathrm{mmol}$ alkyne. 0.5 minol I.jCl. 0.025 mmol Pd source. I minol base. and $10 \mathrm{mI}$. of DMF.

palladium source provided the similar isolated yield, while $\mathrm{Pd}\left(\mathrm{P}^{3} \mathrm{~h}_{3}\right)_{2} \mathrm{Cl}_{2}$ and $\mathrm{Pd}\left(\mathrm{P}^{\mathrm{P}} \mathrm{h}_{3}\right)_{4}$ gave lower yields of the desired products (entries 1-4). We also examined the effect of different bases with Pd(OAc) 2 as a palladium sources. The $\mathrm{Pd}(\mathrm{OAc})_{2}$ was selected due to stability of palladium in reaction medium. The reaction using LiOAc gave the best isolated yields among the examined six bases (entries 4-9). The maximum yield of the desired product was obtained under $5 \mathrm{~mol} \% \mathrm{Pd}(\mathrm{OAc})_{2}, 1$ equivalent of $\mathrm{LiCl}, 2$ equivalents of LiOAc, and 2 equivalents of alkyne in DMr at $110^{\circ} \mathrm{C}$.

The reactions using various 2 -amino-3-iodoquinolines and internal alkynes were examined under optimized reaction conditions to give diverse pyrrolo[2,3-b]quinolines. The results are summarized in lable 2 . Previously, we found that the bulkiness of the substituents on the acetylene and the amine played a major role in determining the regioselectivity of alkyne insertion. The heteroaryl palladium intermediate usually added to the less hindered carbon of the internal alkyne. Heteroannulation using 1-trimethylsilyl alkynes provided highly regioselective products with the trimethylsilyl group next to the nitrogen atom in the pyrrole ring (entries 1-7). By contrast, the reactions using 1-phenyl- 
Table 2. Synthesis of pyrolo[2.3-b]quinolines by palladinn-catalyzed heteroanulation

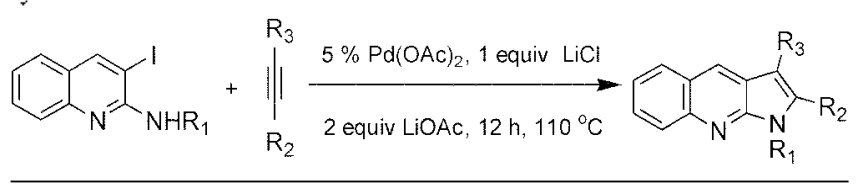

\begin{tabular}{|c|c|c|c|c|}
\hline Entry & $\mathrm{R}_{\mathrm{l}}$ & $\mathrm{R}_{2}$ & $\mathrm{R}_{3}$ & $\begin{array}{l}\text { Isolated yield } \\
\text { (\%) }\end{array}$ \\
\hline 1 & $\mathrm{Bn} 1$ & $\mathrm{Si}\left(\mathrm{CH}_{3}\right)_{3}$ & $\mathrm{CH}_{3}$ & 65 \\
\hline 2 & Ph & $\mathrm{Si}\left(\mathrm{CH}_{3}\right)_{3}$ & $\mathrm{CH}_{s}$ & 67 \\
\hline 3 & $\mathrm{CH}_{3}$ & $\mathrm{Si}\left(\mathrm{CH}_{3}\right)_{3}$ & $\left(\mathrm{CH}_{2}\right)_{3} \mathrm{CH}_{3}$ & 72 \\
\hline 4 & $\mathrm{CH}_{3}$ & $\mathrm{Si}\left(\mathrm{CH}_{3}\right)_{3}$ & $\mathrm{CH}_{2} \mathrm{OH}$ & 63 \\
\hline 5 & $\mathrm{CH}_{3}$ & $\mathrm{Si}\left(\mathrm{CH}_{3}\right)_{3}$ & $\mathrm{CH}_{2} \mathrm{CH}_{2} \mathrm{OH}$ & 60 \\
\hline 6 & $\mathrm{Bn}$ & $\mathrm{Si}\left(\mathrm{CH}_{3}\right)_{3}$ & $\mathrm{Ph}$ & 65 \\
\hline 7 & $\mathrm{Bnn}$ & $\mathrm{Si}\left(\mathrm{CH}_{3}\right)_{3}$ & 3-thiophente & 57 \\
\hline 8 & $\mathrm{Bnn}$ & $\mathrm{CH}_{3}\left(\mathrm{CH}_{2}\right) \Sigma$ & $\mathrm{CH}_{3}\left(\mathrm{CH}_{2}\right)_{2}$ & 74 \\
\hline 9 & $\mathrm{Bnn}$ & $\mathrm{Ph}$ & $\mathrm{Ph}$ & 80 \\
\hline $10^{b}$ & Ph & $\mathrm{Ph}$ & $\mathrm{CH}_{3}$ & $84(2: 1)$ \\
\hline
\end{tabular}

"All reactions were run on a $0.5 \mathrm{mmol}$ scale with $10 \mathrm{~mL}$ of DMF. ${ }^{\text {t T The }}$ isomeric ratio of $R_{2}$ and $R_{3}$ was determined by $H^{3} N M R$ spectroscopy

propyne provided two regio-isomeric products (entry 10). The major isomer had a phenyl substituent next to the nitrogen atom in the pyrrole ring.

\section{Conclusions}

The palladium-catalyzed heteroannulation of 2-amino-3iodoquinoline derivatives with l-trimethylsilyl internal alkynes provided a convenient new route for the synthesis of various 1.2.3-trisubstituted pyrrolo[2.3-b]quinolines. Specially, the heteroannulation provided highly regioselective products with the trimethylsilyl group next to the nitrogen atom in the pyrrole ring. The 2-trimethylsilyl group of py rrolo[2.3- $b$ ]quinolines could be transformed into another functional group to overcome selectivity problem of heteroanunulation in unsymmetric alkynes.

\section{Experimental Section}

The infrared spectra were obtained on Jasco FT-IR 410 spectrometer. All ${ }^{1} \mathrm{H}$ - and ${ }^{13} \mathrm{C}$ NMR Spectra were recorded on a varian $400 \mathrm{MHz}$ spectrometer. Chemical shift are given as value with reference to tetramethylsilane (TMS) as an internal standard. The GC-MS spectra were obtained on a Shimazu QP 1000 GC-MS. Melting points were determined on Mut-TEM apparatus and are uncorrected. Microanalyses were performed by Chungnam national university with $\mathrm{CE}$ Instrument EA 1110. Products were purified by flash chromatography on 230-400 mesh ASTM 60 silicagel. All of bases. $\mathrm{LiCl}$ and palladium species were purchased from Aldrich Chemical $\mathrm{Co}$. The other chemicals were used directly as obtained from commercial sources unless otherwise noted.

\section{Preparation of Starting Materials}

2-Methylamino-3-iodoquinoline ${ }^{14} n$ - $\mathrm{BuLi}(2.5 \mathrm{M}$ in hexane $20 \mathrm{~mL}$. $50 \mathrm{mmol}$ ) was slowly added to a magnetically stirred solution of diisopropylamine $(5.05 \mathrm{~g} .50 \mathrm{mmol})$ in dry THF $(125 \mathrm{~mL})$ under $\mathrm{N}_{2}$ at $-78^{\circ} \mathrm{C}$. The solution of LDA was stirred at $-78^{\circ} \mathrm{C}$ for $1 \mathrm{~h}$. 2-chloroquinoline $(8.2 \mathrm{~g}$. $50 \mathrm{mmol})$ in THF ( $25 \mathrm{~mL}$ ) was added slowly to the reaction mixture at $-78^{\circ} \mathrm{C}$ and stirred for $4 \mathrm{~h}$ at the same temperature at $-78{ }^{\circ} \mathrm{C}$. The iodine solution $(15.2 \mathrm{~g} .50 \mathrm{~mL}$ THF) was slowly added to a solution of lithiated 2-chloroquinoline. The resulting solution was stirred for $2 \mathrm{~h}$ at $-78{ }^{\circ} \mathrm{C}$ and allowed to warm to room temperature over $5 \mathrm{~h}$. After removing the solvent under reduced pressure. the residue was extracted using $\mathrm{Et}_{2} \mathrm{O}$ and decolorized with saturated $\mathrm{NaHSO}_{3}$ aqueous solution. The organic layer was dried over $\mathrm{MgSO}_{4}$. filtered. and concentrated. 2-Chloro-3-iodoquinoline $(10.8 \mathrm{~g} .75 \%)$ was obtained by column chromatography with hexane/ethyl acetate $(10: 1): \mathrm{mp}: 145-146^{\circ} \mathrm{C}: \mathrm{IR}(\mathrm{KBr})$ 3050. 3030. 1610.1575, 1560, 1545, 1485 $\mathrm{cm}^{-1}$. ${ }^{1} \mathrm{H}$ NMR $\left(\mathrm{CDCl}_{3}\right) \delta 8.40$ (s. $\left.1 \mathrm{H} . \mathrm{ArH}\right), 7.90-7.30(\mathrm{~m}, 4 \mathrm{H} . \mathrm{ArH}):{ }^{13} \mathrm{C}$ NMR $\left(\mathrm{CDCl}_{3}\right) \delta 162.7,148.7 .146 .3 .132 .1 .128 .7 .128 .2$. 127.9. 126.8. 84.7: $\mathrm{Ms} \mathrm{m} / \mathrm{z}$ (relative intensity): $289\left(\mathrm{M}^{-}, 38\right)$. 254 (26). 128 (30), $106(48), 91$ (100), 65 (28).

2-Chloro-3-iodo-quinoline ( $1.4 \mathrm{~g} .4 .84 \mathrm{mmol})$. methylamine ( $40 \%$ aqueous solution. $10 \mathrm{~mL}$ ), and ethanol $(10 \mathrm{~mL})$ were added to sealed tube and was reacted at $140^{\circ} \mathrm{C}$ for 10 $\mathrm{h}$. The resulting mixture was extracted using ethyl acetate and water. The organic layer was dried over $\mathrm{MgSO}_{4}$, filtered. and concentrated. 2-Methylamino-3-iodoquinoline ${ }^{\text {ta }}(1.0 \mathrm{~g}$. $73 \%$ ) was obtained by colunn chromatography using hexane/ ethyl acetate (10:1): $\mathrm{mp}: 84-85^{\circ} \mathrm{C}:$ IR $(\mathrm{KBr}) 3420,3040$. 2990. 2950. 2900. 1615. 1595. 1555. $1525 \mathrm{~cm}^{-1}:{ }^{1} \mathrm{H}$ NMR $\left(\mathrm{CDCl}_{3}\right) \delta 8.30$ (s. 1H. ArH). 7.85-7.00 (m. 4H. ArH). 5.25 (brs. $1 \mathrm{H}, \mathrm{NH}$ ), 3.15 (d. $\left.3 \mathrm{H}, J=5.6 \mathrm{~Hz}, \mathrm{~N}-\mathrm{CH}_{3}\right):{ }^{13} \mathrm{C} \mathrm{NMR}$ $\left(\mathrm{CDCl}_{3}\right) \delta 154.2,146.4 .139 .8,130.0,127.1,126.4 .126 .3$. 124.9. 122.4. 29.4: $\mathrm{Ms} \mathrm{m} / \mathrm{z}$ (relative intensity): $284\left(\mathrm{M}^{-}, 38\right)$. 155 (28). 125 (42). 106 (52). 91 (100), 65 (21): Anal. Calc. for $\mathrm{C}_{11} \mathrm{H}_{9} \mathrm{IN}_{2}$ : C. 42.28: H. 3. 19: N. 9.86. Found: C. 42.35: H. 3.17: N. 9.84 .

2-Benzylamino-3-iodoquinoline. This compound was prepared in $75 \%$ yields by the substitution of 2-chloro-3iodo-quinoline with benzylamine: $\mathrm{mp}: 179-180^{\circ} \mathrm{C}$ : IR $(\mathrm{KBr})$ 3397.3025. 1582. $1510.694 \mathrm{~cm}^{-1}:{ }^{1} \mathrm{H} \mathrm{NMR}\left(\mathrm{CDCl}_{3}\right) \delta 8.35$ (s. $1 \mathrm{H}, \mathrm{ArH}$ ), 7.73-7.17 (m. 9H. ArH), 5.55 (br s. $1 \mathrm{H}, \mathrm{NH}$ ). 4.81 (d. $\left.2 \mathrm{H} . J=5.6 \mathrm{~Hz} . \mathrm{ArCH}_{3}\right):{ }^{13} \mathrm{C} \mathrm{NMR}\left(\mathrm{CDCl}_{3}\right) \delta 153.2$. $147.3 .146 .5,129.3,130.1,128.6,127.9 .127 .3,126.4$. $126.3,125.0 .122 .6,83.2,46.4: \mathrm{Ms} \mathrm{m} / \mathrm{z}$ (relative intensity): $360\left(\mathrm{M}^{-}, 36\right) .231$ (29). 128 (28). $116(33) .106(100) .91$ (48). 65 (28): Anal. Calc. for $\mathrm{C}_{16} \mathrm{H}_{13} \mathrm{IN}_{2}:$ C. $53.35: \mathrm{H}, 3.64$ : N. 7.78. Found: C. 53.40: H. 3.60: N. 7.75.

2-Phenylamino-3-iodoquinoline. This compound was prepared in $70 \%$ yield by the substitution of 2-chloro-3iodoquinoline with aniline: $\mathrm{mp}$ : 150-151 ${ }^{\circ} \mathrm{C}$ : IR (KBr) 3305. 3110. 1594. 1480, 908. $700 \mathrm{~cm}^{-1}$ : ${ }^{1} \mathrm{H}$ NMR $\left(\mathrm{CDCl}_{3}\right) \delta 8.43$ (s. $1 \mathrm{H}, \mathrm{ArH}), 7.89-7.07$ (m. $10 \mathrm{H}, \mathrm{ArH}, \mathrm{NH}):{ }^{13} \mathrm{C} \mathrm{NMR}\left(\mathrm{CDCl}_{3}\right)$ $\delta 150.2 .147 .1,146.6,140.0,130.2,128.8 .127 .0,126.2$. 125.5. 123.6. 122.9. 119.5. 83.5: Ms m/z (relative intensity): $345\left(\mathrm{M}^{+} .100\right), 218(54), 109(43)$ : Anal. Calc for $\mathrm{C}_{15} \mathrm{H}_{11} \mathrm{~N}_{2}$ C. 52.04: H. 3.20: N. 8.09. Found: C. 52.11: H. 3.18: N. 8.07.

Trimethyl-thiophen-3-ylethynyl-silane. 3-Iodo-thiophene (2.1 g. $10.0 \mathrm{mmol}$ ), (trimethylsilyl)-acetylene $(1.1 \mathrm{~g} .12 .0$ 
mmol). and $\mathrm{PdCl}_{(}\left(\mathrm{PPh}_{3}\right) \pm(140 \mathrm{mg} .2 \mathrm{~mol} \%)$ were added in $40 \mathrm{~mL}$ of $\mathrm{Et}_{3} \mathrm{~N}$. After stirring the mixture for 5 min. $\mathrm{CuI}(20$ mg. $1 \mathrm{~mol} \%$ ) was added. The resulting solution was heated for $3 \mathrm{~h}$ at $50^{\circ} \mathrm{C}$ under nitrogen atmosphere. The reaction mixture was allowed to cool to room temperature and the ammonium salt was removed by filtration. The solvent was removed under reduced pressure. and residue was purified by column chromatography. Trimethỵl-thiophen-3-y lethỵnylsilane was obtained $90 \%$ yield as a yellow oil: ${ }^{1} \mathrm{H}$ NMR $\left(\mathrm{CDCl}_{3}\right) \delta 7.23-7.18(\mathrm{~m} .2 \mathrm{H} . \mathrm{ArH}) .7 .04(\mathrm{~d} .1 \mathrm{H} . J=8.0 \mathrm{~Hz}$. $\mathrm{ArH}), 0.48$ (s. 9H. $\left.\mathrm{Si}\left(\mathrm{CH}_{3}\right)_{3}\right):{ }^{13} \mathrm{C}$ NMR $\left(\mathrm{CDCl}_{3}\right) \delta 127.9$. 127.3. 126.6, 126.1, 69.9, 62.9, -0.1: Ms $\mathrm{m} / \mathrm{z}$ (relative intensity): $180\left(\mathrm{M}^{+} .100\right), 97(52) .24(14)$.

4-Trimethylsilyl-3-butyn-1-ol. $n$ - $\mathrm{BuLi}$ (2.5 M in hexane. $80 \mathrm{~mL} .0 .2 \mathrm{~mol}$ ) was added in $500 \mathrm{~mL}$ of THF at $-15^{\circ} \mathrm{C} .3-$ Butyn-1-ol (7.0 g. $0.1 \mathrm{~mol})$ was dissolved in $50 \mathrm{~mL}$ of THF and the solution was slowly added to $n$-buty'llithium solution. Subsequently: chlorotrimethylsilane $(24 \mathrm{~g} .0 .2 \mathrm{~mol})$ was introduced over a period of $30 \mathrm{~min}$ with cooling at -15 ${ }^{\circ} \mathrm{C}$. The resulting mixture was stirred for $3 \mathrm{~h}$ at room temperature and poured into $100 \mathrm{~mL}$ of $10 \%$ acetic acid. The reaction mixture was stirred for $\mathrm{lh}$ at room temperature. and neutralized with aqueous sodium bicarbonate. Diethỵl ether was added to the reaction mixture. and organic layer was separated form aqueous layer. The organic layer was dried over magnesium sulfate and filtered. Diethyll ether was removed by evaporation in water bath and the residue was distilled through a $40 \mathrm{~cm}$ Vigreux column. giving the desired alcohol (11 g. 81\%): b.p $90{ }^{\circ} \mathrm{C} / 25 \mathrm{~mm} H \mathrm{Hg}:{ }^{1} \mathrm{H} \mathrm{NMR}\left(\mathrm{CDCl}_{3}\right)$ $\delta 3.64$ (t. $2 \mathrm{H}, J=7.0 \mathrm{~Hz}, \mathrm{CH}_{2} \mathrm{O}$ ). 2.46 (t. $2 \mathrm{H} . J=7.0 \mathrm{~Hz}$. $\mathrm{CH}_{2}$ ). 2.38 (br. $\left.1 \mathrm{H} . \mathrm{OH}\right), 0.12$ (s. $\left.9 \mathrm{H} . \mathrm{Si}\left(\mathrm{CH}_{3}\right)_{3}\right):{ }^{13} \mathrm{C} \mathrm{NMR}$ $\left(\mathrm{CDCl}_{3}\right) \delta 103.4,69.8,60.5,23.8,-0.1: \mathrm{Ms} \mathrm{m} / \mathrm{z}$ (relative intensity): $142\left(\mathrm{M}^{-}, 100\right), 140(42) .125$ (22).

General Procedure of the palladium-catalyzed heteroannulation of internal alkynes. Palladium acetate $(6 \mathrm{mg}$. $0.025 \mathrm{mmol}$ ). $\mathrm{LiCl}$ (22 mg. $0.5 \mathrm{mmol}$ ). LiOAc (66 mg. 1.0 mmol). 2-methy lamino-3-iodoquinoline (142 mg. $0.5 \mathrm{mmol}$ ). l-trimethylsilylpropy'ne (112 mg. $1.0 \mathrm{mmol}$ ) and DMF (10 $\mathrm{mL}$ ) were added to a pressure tube equipped with a stirring bar. After heating the reaction mixture for $10 \mathrm{~h}$ at $110^{\circ} \mathrm{C}$. the resulting solution was diluted with ethyl acetate and washed with saturated aqueous ammonium chloride. The organic layer was dried over $\mathrm{MgSO}_{4}$. filtered. and concentrated. The residue was purified by column chromatography using hexaneethyl acetate. 1.3-Dimethy 1-2-trimethy lsilyl-py'rrolo[2.3-b]quinoline $(100 \mathrm{mg} .75 \%$ ) was obtained as a yellow solid: mp: 86-87 ${ }^{\circ} \mathrm{C}$ : IR $(\mathrm{KBr}) 3059,2950,1606,1568,1441,1245$. $870.843 .749 \mathrm{~cm}^{-1}$. ${ }^{1} \mathrm{H}$ NMR $\left(\mathrm{CDCl}_{3}\right) \delta 8.28$ (s. $1 \mathrm{H} . \mathrm{ArH}$ ). 8.12 (d. $1 \mathrm{H} . J=8.0 \mathrm{~Hz}$. ArH). 7.95 (d. $1 \mathrm{H} . J=8.0 \mathrm{~Hz}, \mathrm{ArH}$ ). 7.66-7.62 (m. 1H. ArH) . 7.41-7.37 (m. 1H. ArH). 4.04 (s. $3 \mathrm{H} . \mathrm{NCH}_{3}$ ). 2.51 (s. $1 \mathrm{H} . \mathrm{ArCH}_{3}$ ). 0.52 (s. $\left.9 \mathrm{H} . \mathrm{Si}\left(\mathrm{CH}_{3}\right)_{3}\right):{ }^{13} \mathrm{C}$ NMR $\left(\mathrm{CDCl}_{3}\right) \delta 151.8,145.7,140.8,128.4,127.7,127.7$. 125.3. 124.0. 123.3. 122.2.118.0. 31.5. 10.9. 1.1: Ms m/z (relative intensity): $268\left(\mathrm{M}^{-}, 100\right), 253(57), 209$ (15), 195 (46). 126 (18): Anal. Calc for $\mathrm{C}_{16} \mathrm{H}_{3} \mathrm{~N}_{2} \mathrm{Si}$. C. $71.59:$ H. 7.51 : N. 10.44. Found: C. 71.64: H. 7.49: N. 10.39. The following compounds were obtained using the above general procedure.
1-Benzyl-3-methyl-2-trimethylsilylpyrrolo[2,3-b]quinoline. This compound was obtained as a yellow solid in $65 \%$ yield from the reaction of 2-benzylamino-3-iodoquinoline with 1trimetylsilyl-1-propy'ne: mp: $111-112{ }^{\circ} \mathrm{C}$ : IR (KBr) 2920. 1605. 1494, $1130,840 \mathrm{~cm}^{-1}:{ }^{1} \mathrm{H}$ NMR $\left(\mathrm{CDCl}_{3}\right) \delta 8.33$ (s. 1H. ArH). 8.02-7.94 (m, 2H, ArH). 7.62-7.54 (m, 1H. ArH). 7.41-7.32 (m. 1H. ArH), 7.26-7.17 (m. 3H. ArH). 6.82-6.78 (m. $2 \mathrm{H}, \mathrm{ArH}$ ), 5.81 (s. $2 \mathrm{H}, \mathrm{ArCH}_{2}$ ). 2.55 (s. $3 \mathrm{H}, \mathrm{ArCH}_{3}$ ). 0.26 (s. 9H. Si $\left.\left(\mathrm{CH}_{3}\right)_{3}\right):{ }^{13} \mathrm{C} \mathrm{NMR}\left(\mathrm{CDCl}_{3}\right) \delta 148.7 .145 .2$. $138.7 .128 .3,127.5,127.4,127.2 .126 .8,125.8,124.9$. $124.4,121.5,120.8 .116 .7,94.7 .46 .0,10.4,0.3: \mathrm{Ms} \mathrm{m} / \mathrm{z}$ (relative intensity): $344\left(\mathrm{M}^{+} .100\right), 271(22), 253(50), 221$ (40). 207 (35). 195 (24). 91 (86). 73 (31). 59 (35): Anal. Calc. for $\mathrm{C}_{2} \mathrm{H}_{2+} \mathrm{N}_{2} \mathrm{Si}$ : C. 76.70: H. 7.02: N. 8.13. Found: $\mathrm{C}$. 76.75: H. 7.04: N. 8.10.

3-Methyl-1-phenyl-2-trimethylsilylpyrrolo[2,3-b]quinoline. This compound was obtained as a yellow solid in $67 \%$ yield from the reaction of 2-phenylamino-3-iodoquinoline with l-trimetylsilyl-1-propyne: mp: $117-118^{\circ} \mathrm{C}$ : IR $(\mathrm{KBr})$ 2925. 1632.1536. 1451.1138. $836 \mathrm{~cm}^{-1}$ : ${ }^{1} \mathrm{H}$ NMR $\left(\mathrm{CDCl}_{3}\right)$ $\delta 8.32$ (s. 1H. ArH). 7.98-7.93 (m. 2H. ArH), 7.57-7.44 (m. $6 \mathrm{H}, \mathrm{ArH}$ ). $7.38-7.36(\mathrm{~m}, 1 \mathrm{H}, \mathrm{ArH}) .2 .52$ (s. $\left.3 \mathrm{H}, \mathrm{ArCH}_{3}\right) .0 .14$ (s. $\left.9 \mathrm{H} . \mathrm{Si}\left(\mathrm{CH}_{3}\right)_{3}\right):{ }^{13} \mathrm{C}$ NMR $\left(\mathrm{CDCl}_{3}\right) \delta 146.0,142.0,139.8$. $129.6 .129 .3,129.0,128.4,128.2,128.0,127.6,125.3,124.4$. 123.4. 122.7. 119.9. 11.3. 0.7: Ms m/z (relative intensity): 330 $\left(\mathrm{M}^{-}, 100\right) .315(67), 285(27), 257(17), 239(40), 150(65)$. 91 (14), 73 (24), 43 (41): Anal. Calc. for $\mathrm{C}_{21} \mathrm{H}_{22} \mathrm{~N}_{2} \mathrm{Si}$ : C. 76.32: H. 6.71: N. 8.48. Found: C. 76.36: H. 6.74: N. 8.45.

3-Butyl-1 - methyl-2-trimethylsilylpyrrolo[2,3-b]quinoline This compound was obtained as a yellow solid in $72 \%$ yield from the reaction of 2-metlylamino-3-iodoquinoline with 1-trimetylsilyl-1-hexyne: mp: $96-97^{\circ} \mathrm{C}: \mathrm{IR}(\mathrm{KBr}) 3048$. 2950. 1610, 1553, 1440.1238.870. $752 \mathrm{~cm}^{-1}$ : ${ }^{1} \mathrm{H}$ NMR $\left(\mathrm{CDCl}_{3}\right) \delta 8.25$ (s. $\left.1 \mathrm{H} . \mathrm{ArH}\right), 8.11$ (d. $\left.1 \mathrm{H} . J=8.0 \mathrm{~Hz} . \mathrm{ArH}\right)$. 7.97 (d. $1 \mathrm{H} . J=8.0 \mathrm{~Hz}$. ArH). 7.68-7.63 (m. 1H. ArH). 7.427.35 (m. lH, ArH) 4.01 (s. $3 \mathrm{H}, \mathrm{NCH}_{3}$ ), 2.51 (t. $2 \mathrm{H} . J=6.8$ Hz. $\left.\mathrm{ArCH}_{2} \mathrm{CH}_{2}-\right)$, 1.58-1.44 (m. 2H. $\mathrm{ArCH}_{2} \mathrm{CH}_{2}-$ ). 1.05-0.96 (m. $2 \mathrm{H},-\mathrm{CH}_{2}$ ), 0.84 (t. $\left.3 \mathrm{H}, J=7.2 \mathrm{~Hz},-\mathrm{CH}_{2} \mathrm{CH}_{3}\right), 0.07$ (s. $\left.9 \mathrm{H} . \mathrm{Si}\left(\mathrm{CH}_{3}\right)_{3}\right):{ }^{13} \mathrm{C} \mathrm{NMR}\left(\mathrm{CDCl}_{3}\right) \delta 150.3 .145 .7 .140 .8$. $128.6,127.6,127.6,125.3 .123 .9,123.3,122.4,117.8,32.4$. 18.7. 14.6. 10.1. 9.0. 1.0. $\mathrm{Ms} \mathrm{m} / \mathrm{z}$ (relative intensity): 310 $\left(\mathrm{M}^{-}, 100\right) .281$ (46). 253 (25). 209 (43). 195 (21). 126 (16): Anal. Calc. for $\mathrm{C}_{19} \mathrm{H}_{56} \mathrm{~N}_{2} \mathrm{Si}:$ C. 73.49: H. 8.44: N. 9.02 . Found: C. $73.46:$ H. 8.40: N. 9.08 .

(1-Methyl-2-trimethylsilanyl-pyrrolo[2,3-b]quinolin-3yl)-methanol. This compound was obtained as a yellow solid in $63 \%$ yield from the reaction of 2-metlylamino-3iodoquinoline with 3-trimetylsilyl-2-propyl-1ol: $\mathrm{mp}$ : 81-82 ${ }^{\circ} \mathrm{C}$ : IR $(\mathrm{KBr}) 3305,3061,2940,1615,1460,1248,870,740$ $\mathrm{cm}^{-1}$ : ${ }^{1} \mathrm{H}$ NMR $\left(\mathrm{CDCl}_{3}\right) \delta 8.26$ (s. lH. ArH). 8.14 (d. lH. $J$ $=8.0 \mathrm{~Hz}, \mathrm{ArH}) .8 .01(\mathrm{~d} .1 \mathrm{H}, J=8.0 \mathrm{~Hz}, \mathrm{ArH}) .7 .69-7.64(\mathrm{~m}$. 1H. $\mathrm{ArH}$ ). 7.43-7.39 (m. 1H. ArH), 4.75 (s. $2 \mathrm{H} . \mathrm{ArC}_{2} \mathrm{OH}$ ). 4.01 (s. $3 \mathrm{H}, \mathrm{NCH}_{3}$ ). 0.55 (s. $\left.9 \mathrm{H}, \mathrm{Si}\left(\mathrm{CH}_{3}\right)_{3}\right):{ }^{13} \mathrm{C} \mathrm{NMR}$ $\left(\mathrm{CDCl}_{3}\right) \delta 152.1,145.7 .140 .8 .128 .5,127.9,127.7 .125 .4$. $124.4,123.3,123.0,118.2,59.8,33.3,1.0 \mathrm{gs} \mathrm{m} / \mathrm{z}$ (relative intensity): $284\left(\mathrm{M}^{+} .100\right) .253$ (61). 209 (25). 195 (41). 126 (23): Anal. Calc. for $\mathrm{C}_{16} \mathrm{H}_{30} \mathrm{~N}_{2} \mathrm{OSi}$ : C. 67.56: H. 7.09: N. 
9.85. Found: C. 67.60: H. 7.13: N. 9.81 .

2-(1-Methyl-2-trimethylsilanyl-pyrrolo[2,3-b]quinolin3-yl)-ethanol. This compound was obtained as a yellow solid in $60 \%$ yield from the reaction of 2-methylamino-3iodoquinoline with 4-trimethylsilyl-3-butyn-1-ol: mp: 87-88 ${ }^{\circ} \mathrm{C}:$ IR (KBr) 3310. 3065, 2890, 1632, 1520. 1252.867.788. $735 \mathrm{~cm}^{-\mathrm{l}}$ : ${ }^{1} \mathrm{H}$ NMR $\left(\mathrm{CDCl}_{3}\right) \delta 8.24$ (s. $\left.1 \mathrm{H} . \mathrm{ArH}\right) .8 .15$ (d. $1 \mathrm{H} . J=8.0 \mathrm{~Hz}, \mathrm{ArH}) .7 .98(\mathrm{~d} .1 \mathrm{H} . J=8.0 \mathrm{~Hz}, \mathrm{ArH}) .7 .70-$ 7.66 (m. 1H. ArH). 7.42-7.39 (m. 1H. ArH). 4.68-4.63 (m. 2H. $\left.\mathrm{ArCH}_{2} \mathrm{CH}_{3} \mathrm{OH}\right), 4.05$ (s. $\left.3 \mathrm{H} . \mathrm{NCH}_{3}\right) .2 .71$ (t. $2 \mathrm{H} . J=7.0$ $\mathrm{Hz}, \mathrm{ArCH}_{2} \mathrm{CH}_{2} \mathrm{OH}$ ). 0.51 (s. $\left.9 \mathrm{H}, \mathrm{Si}\left(\mathrm{CH}_{3}\right)_{3}\right):{ }^{13} \mathrm{C}$ NMR $\left(\mathrm{CDCl}_{3}\right) \bar{\delta} 152.2,145.8,140.8,128.6,128.0,127.7,125.4$. 124.4. 123.3, 122.8, 118.3, 58.5, 33.2, 23.6. 1.1: Ms m/z (relative intensity): $298\left(\mathrm{M}^{-}, 57\right), 253$ (100), 209 (31), 195 (51). 126 (18): Anal. Calc. for $\mathrm{C}_{17} \mathrm{H}_{32} \mathrm{~N}_{2} \mathrm{OSi}$ : C. 68.58: $\mathrm{H}$. 7.43: N. 9.39. Found: C. 68.63: H. 7.41: N. 9.36

1-Benzyl-3-phenyl-2-trimethylsilylpyrrolo[2,3- $b]$ quinoline. This compound was obtained as a yellow solid in $65 \%$ yield from the reaction of 2-benzylamino-3-iodoquinoline with l-trimethy lphenyl acety lene: mp: $114-115^{\circ} \mathrm{C}: \mathrm{IR}(\mathrm{KBr})$ 3029, 2927, 1602, 1570, 1429, 1249,845, $723 \mathrm{~cm}^{-1}:{ }^{1} \mathrm{H}$ NMR $\left(\mathrm{CDCl}_{3}\right) \delta 8.19$ (s. $\left.1 \mathrm{H} . \mathrm{ArH}\right) .8 .08(\mathrm{~d} .1 \mathrm{H} . J=8.0 \mathrm{~Hz}$. $\mathrm{ArH}) .7 .85$ (d. 1H. $J=8.0 \mathrm{~Hz}, \mathrm{ArH}) .7 .62-7.14(\mathrm{~m}, 10 \mathrm{H}$. $\mathrm{ArH}$ ). 6.95-6.93 (m, 2H, ArH). 5.94 (s. 2H, $\mathrm{ArCH}_{2}$ ). 0.03 (s. 9H. $\left.\mathrm{Si}\left(\mathrm{CH}_{3}\right)_{3}\right):{ }^{13} \mathrm{C}$ NMR $\left(\mathrm{CDCl}_{3}\right) \delta 145.8 .142 .0 .139 .1$. 136.0. 131.8. 131.1. 129.2. 128.5. 128.5. 128.4. 128.3. 128.1. $127.8,127.3,126.9,126.8,126.1,124.8,122.7,47.2,0.8 \mathrm{Ms}$ $\mathrm{m} / \mathrm{z}$ (relative intensity): $406\left(\mathrm{M}^{-}, 100\right) .333(54) .315(34), 216$ (20). $91(67) .73(44), 65(17)$. Anal. Calc. for $\mathrm{C}_{27} \mathrm{H}_{36} \mathrm{~N}_{2} \mathrm{Si}$ : C. $79.76:$ H. 6.45: N 6.89 . Found: C. 79.82: H. 6.44: N 6.92 .

1-Benzyl-3-thiophen-3-y]-2-trimethylsilylpyrrolo[2,3-b]quinoline. This compound was obtained as a yellow solid in $57 \%$ yield from the reaction of 2-benzylamino-3-iodoquinoline with trimethyl-thiophen-3-y lethynyl-silane: mp: $151-$ $152^{\circ} \mathrm{C}: \mathrm{IR}(\mathrm{KBr}) 3026,2922.1598,1562,1436,1240,747$. $718 \mathrm{~cm}^{-1}$ : ${ }^{1} \mathrm{H}$ NMR $\left(\mathrm{CDCl}_{3}\right) \delta 8.21$ (s. $1 \mathrm{H} . \mathrm{ArH}$ ). 8.05 (dd. $1 \mathrm{H}, J=8.2 .0 .6 \mathrm{~Hz}, \mathrm{ArH}) .7 .89$ (d. $1 \mathrm{H} . J=8.2 \mathrm{~Hz}, \mathrm{ArH})$. 7.66-7.15 (m. 8H. ArH). 6.96-6.92 (m. 2H. ArH). 5.91 (s. 2H. $\left.\mathrm{ArCH}_{2}\right), 0.08$ (s.9H, $\left.\mathrm{Si}\left(\mathrm{CH}_{3}\right)_{3}\right):{ }^{13} \mathrm{C} \mathrm{NMR}\left(\mathrm{CDCl}_{3}\right) \delta$ $150.9,145.3,141.8,138.5,135.3,129.8,127.8,127.7$. 127.4. $127.3,126.3,125.8,125.5,124.4,124.2,123.6$. 122.2. 122.0, 120.2,46.4. $0.5: \mathrm{Ms} \mathrm{m} / \mathrm{z}$ (relative intensity): $412\left(\mathrm{M}^{+}, 100\right), 328(20) .253(48) .221$ (38), $207(36), 195$ (22). 91 (75). 59 (18): Anal. Calc. for $\mathrm{C}_{2 \leq} \mathrm{H}_{24} \mathrm{~N}_{2} \mathrm{SSi}$ : C. 72.77: H. 5.86: N. 6.79. Found: C. 72.82: H. 5.79: N 6.78.

1-Benzyl-2,3-dipropylpyrrolo[2,3- $b]$ quinoline. This compound was obtained as a yellow solid in $74 \%$ yield from the reaction of 2-benzylamino-3-iodoquinoline with 4octyne: mp: $129-130^{\circ} \mathrm{C}$ : IR (KBr) $2959,2870,1486,1242$. $753 \mathrm{~cm}^{-1}$ : ${ }^{1} \mathrm{H}$ NMR $\left(\mathrm{CDCl}_{3}\right) \delta 8.17$ (s. $\left.1 \mathrm{H} . \mathrm{ArH}\right) .8 .09$ (d. $1 \mathrm{H} . J=8.2 \mathrm{~Hz}, \mathrm{ArH}) .7 .88$ (d. $1 \mathrm{H}, J=8.2 \mathrm{~Hz} . \mathrm{ArH}) .7 .65-$ $7.58(\mathrm{~m}, 1 \mathrm{H}, \mathrm{ArH}), 7.41-7.35$ (m, 1H, ArH) $7.25-7.15(\mathrm{~m}, 5 \mathrm{H}$. $\mathrm{ArH}) .5 .70$ (s. 1H. $\mathrm{ArCH}_{2}$ ). 2.84-2.71 (m, 2H. $\mathrm{ArCH}_{2} \mathrm{CH}_{2}$ ). 1.76-1.61 (m. 2H, $\mathrm{ArCH}_{2} \underline{\mathrm{CH}}_{2}-$ ). 1.06-0.95 (m. 3H. $\left.-\overline{\mathrm{CH}}_{2} \mathrm{CH}_{3}\right)$ ): ${ }^{13} \mathrm{C} \mathrm{NMR}\left(\mathrm{CDCl}_{\mathfrak{j}}\right) \delta 150.2,144.8,137.9,128.4,128.2 .128 .0$. $127.9 .127 .4,127.2,124.5,124.4,123.4,122.1,114.9 .92 .8$. $53.5,26.7,26.6,25.6,24.7,13.3,13.0 . \mathrm{Ms} \mathrm{m} / \mathrm{z}$ (relative intensity): $342\left(\mathrm{M}^{+} .75\right) .299$ (16). 256 (39). $165(20) .154$ (15). 127 (13). 91 (100). 65 (34): Anal. Calc. for $\mathrm{C}_{24} \mathrm{H}_{26} \mathrm{~N}_{2}$ : C. 84.17: H. 7.65: N. 8.18. Found: C. 84.12: H. 7.68: N 8.20.

1-Benzy]-2,3-diphenylpyrrolo[2,3-b]quinoline. This compound was obtained as a yellow solid in $80 \%$ y ield from the reaction of 2-benzlamino-3-iodoquinoline with diphenylacetylene: mp: $219-220^{\circ} \mathrm{C}$ : IR (KBr) 3050. 1600. 1569. 1422, 1387, $788 \mathrm{~cm}^{-1}:{ }^{l} \mathrm{H}$ NMR $\left(\mathrm{CDCl}_{3}\right) \delta 8.52(\mathrm{~s}$. 1H. ArH), 8.14 (d. $1 \mathrm{H}, J=8.4 \mathrm{~Hz}, \mathrm{ArH}), 7.92$ (d. $1 \mathrm{H}, J=8.4$ Hz. ArH), 7.64-7.61 (m. 2H. ArH), 7.41-6.98 (m. 15H. $\mathrm{ArH}), 5.60$ (s. $2 \mathrm{H}, \mathrm{ArCH}) ;{ }^{13} \mathrm{C}$ NMR $\left(\mathrm{CDCl}_{3}\right) \delta 150.2$ $145.5,141.4,138.5,134.1,131.6,131.2,130.9,129.6$. 128.6. 128.4, 128.3, 128.3, 128.2, 128.1, 127.7, 127.3. $127.0,126.3,126.0,125.4,122.9,122.0,46.0: \mathrm{Ms} \mathrm{m} / \mathrm{z}$ (relative intensity): $410\left(\mathrm{M}^{+} .100\right), 333(41), 317(60), 214$ (11). 166 (17). 91 (77), 65 (18): Anal. Calc. for $\mathrm{C}_{30} \mathrm{H}_{22} \mathrm{~N}_{2}: \mathrm{C}$. 87.77: H. 5.40: N, 6.82. Found: C. 87.73: H. 5.42: N 6.85.

Acknowledgments. This work was supported by Korea Research Foundation Grant (KRF-2001-015-DP0330).

\section{References}

1. Katritzhy, R: Rees. C. W. Comprehensive Heterocyclic Chemistry, Pergamon Press: 1984: Vol 4. pp 988-992. and references cited therein.

2. (a) Khan. M. A.: Da Rocha. T. F. Heterocycles 1977. 6. 1229 and references cited therein. (b) Smith, C. D.: Latrence, D. S. WO 01: 74790. 2001. (c) Fukuda, Y.: Tanioka, A. WO 0006571.2000.

3. Stringer, M. B.; Candeloro, V; Bowie, J. H.: Prager, R. H.: Engelhardt. L. M.: White. A. H. $J$. Chem Soc. Perkin Trans I 1984.2529.

4. Zimmer. H.: Ambruster. D. C.: Khridia. S. P.: Lankin. D. C Tetrahedron Lett. $1969,+6.4053$

5. (a) Shanmugam, P. Thiruvengadam, T. K.: Ramakrishnan, V. T: Sunthesis 1976. 393. (b) Murugesan. M: Soundarajan. N: Ramasamy: K.: Shanmugam. P. Snthesis 1979.352.

6. Himbert. G.: Schwickerath. W: Maas. G. Leibigs Am. Chent. 1985. 1398.

7. Davis, M. L.: Wakefield. B. J.: Wardell, J. A. Tetrohedron 1992. 18. 939 .

8. Molina. P; Alcantra, J.: Lopez-Leonardo, C. Tetrahedron 1997. 53. 3281

9. Tanaka. T.: Iwakuma. T.: Wagatsuma. M.: Iijima. I. J. Heterock dic Chent 1972.9.1355.

10. (a) Larock. R. C. Yum. E. K. J. Am. Chem. Soc. 1991, 113. 6689 (b) Larock, R. C., Yum. E. K.: Reffik, D. .l. Org. Chem. 1998. 63. 7652 .

11. (a) Larock. R. C. J. Organomet. Chem. 1999. 576.111 . (b) Larock. R. C. Pure and .Appl. Chem. 1999. 71. 1435. (c) Li. J. T.: Gribble. G. W. Palladim in Heterocuctic Chemistiy. Pergamon: 2000: $\mathrm{pp} 143-146$.

12. (a) Park. S. S.: Choi, J.-K.; Yum, E. K; Ha. D.-C. Tetrahedron Lett. 1998, 39.627. (b) Chi. S. M.: Choi, J.-K.: Yum. E. K.: Chi. D. Y. Tetahedron. Lett. 2000. 11.919. (c) Lee. M. S.: Yum. E. K. Bull. Korean Chem. Soc. 2002. 23.535.

13. (a) Kang. S. K.: Park. S. S.: Kim. S. S.: Choi. T.-K.: Yumn. E. K Tetrahedhon Lett. 1999, 40. 4379. (b) Yum, E. K.; Kang, S. S: Kim. S. S.: Choi. J.-K.: Cheon. H. G. Bioorg Med. Chem. Lett. 1999. 9. 1819. (c) Kim. S. S.; Cheon. H. G.; Kang. S. K.; Yum, E. K.: Choi. T.-K. Heterocycles 1998. 48.221

14. (a) Marsais. F.: Gogard. A.: Queguiner. G. J. Heterocklic Chent. 1989. 26. 1589. (b) Queguiner. G. J. Heterocwic Chent. 2000. 37. 615. 